QUESTIONNAIRES (English)

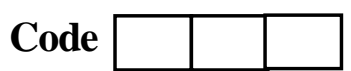

\begin{tabular}{|c|c|c|c|}
\hline \multicolumn{4}{|c|}{ Socioeconomic factors } \\
\hline Age & years & $\square$ Male & $\square$ female \\
\hline \multicolumn{4}{|c|}{ Township } \\
\hline \multicolumn{4}{|c|}{ Education } \\
\hline & $\square$ Illiterate & $\square$ Middle school education level & \\
\hline & $\square$ Read and write & $\square$ High school education level & \\
\hline & $\square$ Primary school education level & $\square$ Graduate and above & \\
\hline \multicolumn{4}{|c|}{ Occupation } \\
\hline & \multicolumn{3}{|l|}{$\square$ Dependent } \\
\hline & \multicolumn{3}{|l|}{$\square$ Unskilled laborer } \\
\hline & \multicolumn{3}{|l|}{$\square$ Own business } \\
\hline & \multicolumn{3}{|l|}{$\square$ Private employee } \\
\hline & $\square$ Government staff & & \\
\hline
\end{tabular}

Marital status

$\square$ Single

Married

Separate

Divorced

Widowed

Living situation

Alone

$\square$ With family

With friends

Others, please specify

\begin{tabular}{|l|ll|}
\hline Household size & Monthly family income & kyats \\
\hline Smoking status & $\square$ Yes & $\square$ No \\
\hline Alcohol drinking & $\square$ Yes & $\square$ No \\
\hline Comorbidity & $\square$ Yes & $\square$ No \\
\hline
\end{tabular}




\begin{tabular}{|lll|}
\hline Epidemic-related factors & & \\
\hline Contact history & $\square$ Yes & $\square$ No \\
\hline Infected to family members & $\square$ Yes & $\square$ No \\
\hline Travelling history to abroad & $\square$ Yes & $\square$ No \\
\hline $\begin{array}{l}\text { Travelling history to townships under stay } \\
\text { at home order }\end{array}$ & $\square$ Yes & $\square$ No \\
\hline Presenting symptom & $\square$ Yes & $\square$ No \\
\hline Psychological factors & & $\square$ No \\
\hline Psychological history & $\square$ Yes & $\square$ No \\
\hline Psychological history in family members & $\square$ Yes & $\square$ No \\
\hline Psychosocial supports & & Yes \\
\hline Social support & $\square$ Yes & \\
\hline Mental support & & \\
\hline
\end{tabular}




\section{Depressive symptoms in patients with COVID-19}

Below is a list of the ways you might have felt or behaved. Please tell me how often you have felt this way during the past week.

\begin{tabular}{|c|c|c|c|c|c|}
\hline \multirow{2}{*}{\multicolumn{2}{|c|}{ Statements }} & \multicolumn{4}{|c|}{ During the past week } \\
\hline & & 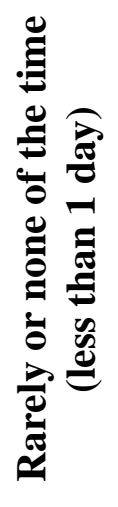 & 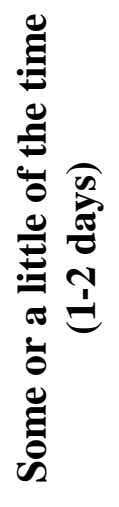 & 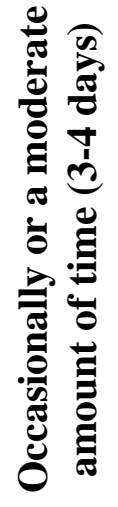 & 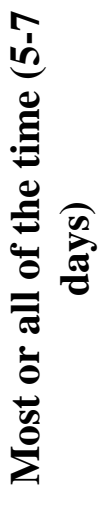 \\
\hline 1 & I was bothered by things that usually don't bother me. & $\square$ & $\square$ & $\square$ & $\square$ \\
\hline 2 & I did not feel like eating; $m$ appetite was poor. & $\square$ & $\square$ & $\square$ & $\square$ \\
\hline 3 & $\begin{array}{l}\text { I felt that I could not shake off the blues even with } \\
\text { help from my family or friends. }\end{array}$ & $\square$ & $\square$ & $\square$ & $\square$ \\
\hline 4 & I felt I was just as good as other people. & $\square$ & $\square$ & $\square$ & $\square$ \\
\hline 5 & I had trouble keeping my mind on what I am doing. & $\square$ & $\square$ & $\square$ & $\square$ \\
\hline 6 & I felt depressed. & $\square$ & $\square$ & $\square$ & $\square$ \\
\hline 7 & I felt that everything I did was an effort. & $\square$ & $\square$ & $\square$ & $\square$ \\
\hline 8 & I felt hopeful about the future. & $\square$ & $\square$ & $\square$ & $\square$ \\
\hline 9 & I thought my life had been a failure. & $\square$ & $\square$ & $\square$ & $\square$ \\
\hline 10 & I felt fearful. & $\square$ & $\square$ & $\square$ & $\square$ \\
\hline 11 & My sleep was restless. & $\square$ & $\square$ & $\square$ & $\square$ \\
\hline 12 & I was happy. & $\square$ & $\square$ & $\square$ & $\square$ \\
\hline
\end{tabular}


13 I talked less than usual.

14 I felt lonely.

15 People were unfriendly.

16 I enjoyed life.

17 I had crying spells.

18 I felt sad.

19 I felt people dislike me.

20 I could not get "going".

$\begin{array}{llll}\square & \square & \square & \square \\ \square & \square & \square & \square \\ \square & \square & \square & \square \\ \square & \square & \square & \square \\ \square & \square & \square & \square \\ \square & \square & \square & \square \\ \square & \square & \square & \square \\ \square & \square & \square & \square\end{array}$

